\title{
Strange baryon spectroscopy in the relativistic quark model
}

\author{
R. N. Faustov and V. O. Galkin \\ Dorodnicyn Computing Center, Federal Research Center "Computer Science and Control", \\ Russian Academy of Sciences, Vavilov Str. 40, 119333 Moscow, Russia
}

\begin{abstract}
Mass spectra of strange baryons are calculated in the framework of the relativistic quark model based on the quasipotential approach. Baryons are treated as the relativistic quark-diquark bound systems. It is assumed that two quarks with equal constituent masses form a diquark. The diquark excitations and its internal structure are consistently taken into account. Calculations are performed up to rather high orbital and radial excitations of strange baryons. On this basis the Regge trajectories are constructed. The obtained results are compared with available experimental data and previous predictions. It is found that all masses of the 4- and 3-star, as well as most of the 2- and 1-star states of strange baryons with established quantum numbers are well reproduced. The developed relativistic quark-diquark model predicts less excited states than three-quark models of strange baryons.

PACS numbers: 14.20.Jn, 12.39.Ki, 12.39.Pn
\end{abstract}

\section{INTRODUCTION}

At present the extensive evidence (including lattice calculations) of the existence of diquark correlations in hadrons was collected. ${ }^{1}$ It continues constantly growing with the accumulation of new data on various properties of light and heavy hadrons [1]. Thus recently several charged charmonium- and bottomonium-like states were discovered [1, 2]. They should be inevitably multiquark, at least four quark - tetraquark, states. One of the most successful pictures of such tetraquark states is the diquark-antidiquark model [3, 4]. In the light meson sector it has been argued for a long time that mesons forming the inverted lightest scalar nonet can be well described as tetraquarks [5] treated as diquark-antidiquark bound states [6, 7]. In the baryon sector it is well known that the number of observed excited states both in the light and heavy sectors is considerably lower than the number of excited states predicted in the three-quark picture [8-11]. The introduction of diquarks significantly reduces this number of baryon states since in such a picture some of degrees of freedom are frozen and thus the number of possible excitations is substantially smaller.

In our previous papers we developed the relativistic quark-diquark model of doubly heavy [12] and heavy baryons [13, 14]. We assumed that two heavy quarks in a doubly heavy baryon and two light quarks in a heavy baryon form a diquark. The relativistic quasipotential equation with the QCD-motivated quark-quark interaction was solved for obtaining diquark characteristics, such as the diquark masses and form factors. The calculation of the diquark form factors is necessary for taking into account the diquark internal structure. For doubly heavy diquarks [12] we considered both ground and excited states, while for light diquarks [13] we limited ourselves by only ground state scalar and axial vector diquarks. Then the

\footnotetext{
${ }^{1}$ A vast literature on this subject is available. Therefore we mostly refer to the recent reviews where the references to earlier review and original papers can be found.
} 
baryon masses were calculated by solving the relativistic quark-diquark equation. It was found that the heavy baryon spectra are well described in the proposed approach [13, 14]. The calculated baryon wave functions were used for the description of weak decays of the doubly heavy and heavy baryons in Refs. [15, 16].

Here we extend our relativistic quark-diquark model for the calculation of the mass spectra of strange baryons. These baryons are considered as the bound systems of a quark and diquark, where we assume that a diquark is composed from quarks of the same constituent mass. Thus $\Lambda$ and $\Sigma$ baryons contain the strange $s$ quark and the light $q q(q=u, d)$ diquark, while $\Xi$ and $\Omega$ baryons contain the light $q$ or strange $s$ quark and the strange $s s$ diquark. Our analysis of the strange baryon spectroscopy shows that it is necessary to consider both ground and excited states of these diquarks. As the result the number of obtained baryon states is increased, however it is still significantly less than in the three-quark approaches. The differences become evident for the higher quark excitations in a baryon. Our goal is to calculate the strange baryon spectra up to rather high orbital and radial excitations. On this basis the Regge trajectories for these baryons can be constructed and their linearity can be tested. Moreover, the comparison of the Regge trajectory slopes for strange and charmed baryons as well as light mesons can be made.

The paper is organized as follows. In Sec. II we briefly describe our relativistic quarkdiquark model of baryons. Expressions for the quasipotentials of the quark-quark interaction in a diquark and the quark-diquark interaction in a baryon are given which include both the spin-independent and spin-dependent relativistic contributions. Masses and form factor parameters of ground and excited states of diquarks are calculated. In Sec. III the mass spectra of strange baryons are considered. The obtained results are confronted with available experimental data and predictions of other approaches. We calculate the strange baryon masses up to rather high orbital and radial excitations in the quark-diquark bound system. This allows us to construct their Regge trajectories which are presented in Sec. IV] The corresponding slopes and intercepts are determined. Finally, we give our conclusions in Sec. V.

\section{RELATIVISTIC QUARK-DIQUARK MODEL}

For the calculations of the strange baryon spectra we employ the quasipotential approach and quark-diquark picture of baryons which was previously used for the investigation of the heavy baryon spectroscopy [13, 14]. In our present analysis we closely follow these considerations. The interaction of two quarks in a diquark and the quark-diquark interaction in a baryon are described by the diquark wave function $\Psi_{d}$ of the bound quark-quark state and by the baryon wave function $\Psi_{B}$ of the bound quark-diquark state respectively, which satisfy the quasipotential equation of the Schrödinger type [17]

$$
\left(\frac{b^{2}(M)}{2 \mu_{R}}-\frac{\mathbf{p}^{2}}{2 \mu_{R}}\right) \Psi_{d, B}(\mathbf{p})=\int \frac{d^{3} q}{(2 \pi)^{3}} V(\mathbf{p}, \mathbf{q} ; M) \Psi_{d, B}(\mathbf{q}),
$$

where the relativistic reduced mass is

$$
\mu_{R}=\frac{M^{4}-\left(m_{1}^{2}-m_{2}^{2}\right)^{2}}{4 M^{3}},
$$

and $M$ is the bound state mass (diquark or baryon), $m_{1,2}$ are the masses of quarks ( $q_{1}$ and $q_{2}$ ) which form the diquark or of the diquark $(d)$ and quark $(q)$ which form the baryon $(B)$, 
and $\mathbf{p}$ is their relative momentum. In the center of mass system the relative momentum squared on mass shell reads

$$
b^{2}(M)=\frac{\left[M^{2}-\left(m_{1}+m_{2}\right)^{2}\right]\left[M^{2}-\left(m_{1}-m_{2}\right)^{2}\right]}{4 M^{2}} .
$$

The kernel $V(\mathbf{p}, \mathbf{q} ; M)$ in Eq. (11) is the quasipotential operator of the quark-quark or quark-diquark interaction which is constructed with the help of the off-mass-shell scattering amplitude, projected onto the positive energy states. We assume that the effective interaction is the sum of the usual one-gluon exchange term and the mixture of long-range vector and scalar linear confining potentials, where the vector confining potential contains the Pauli term. The details can be found in Refs. [13, 14]. The resulting quasipotentials are given by the following expressions.

(a) Quark-quark $(q q)$ interaction in the diquark

$$
V(\mathbf{p}, \mathbf{q} ; M)=\bar{u}_{1}(p) \bar{u}_{2}(-p) \mathcal{V}(\mathbf{p}, \mathbf{q} ; M) u_{1}(q) u_{2}(-q),
$$

with

$$
\mathcal{V}(\mathbf{p}, \mathbf{q} ; M)=\frac{1}{2}\left[\frac{4}{3} \alpha_{s} D_{\mu \nu}(\mathbf{k}) \gamma_{1}^{\mu} \gamma_{2}^{\nu}+V_{\text {conf }}^{V}(\mathbf{k}) \Gamma_{1}^{\mu}(\mathbf{k}) \Gamma_{2 ; \mu}(-\mathbf{k})+V_{\text {conf }}^{S}(\mathbf{k})\right],
$$

(b) Quark-diquark $(q d)$ interaction in the baryon

$$
\begin{aligned}
V(\mathbf{p}, \mathbf{q} ; M)= & \frac{\left\langle d(P)\left|J_{\mu}\right| d(Q)\right\rangle}{2 \sqrt{E_{d}(p) E_{d}(q)}} \bar{u}_{q}(p) \frac{4}{3} \alpha_{s} D_{\mu \nu}(\mathbf{k}) \gamma^{\nu} u_{q}(q) \\
& +\psi_{d}^{*}(P) \bar{u}_{q}(p) J_{d ; \mu} \Gamma_{q}^{\mu}(\mathbf{k}) V_{\text {conf }}^{V}(\mathbf{k}) u_{q}(q) \psi_{d}(Q) \\
& +\psi_{d}^{*}(P) \bar{u}_{q}(p) V_{\text {conf }}^{S}(\mathbf{k}) u_{q}(q) \psi_{d}(Q),
\end{aligned}
$$

where $\alpha_{s}$ is the QCD coupling constant, $\left\langle d(P)\left|J_{\mu}\right| d(Q)\right\rangle$ is the vertex of the diquark-gluon interaction which takes into account the diquark internal structure and $J_{d ; \mu}$ is the effective long-range vector vertex of the diquark. The diquark momenta are $P=\left(E_{d}(p),-\mathbf{p}\right), Q=$ $\left(E_{d}(q),-\mathbf{q}\right)$ with $E_{d}(p)=\sqrt{\mathbf{p}^{2}+M_{d}^{2}} . D_{\mu \nu}$ is the gluon propagator in the Coulomb gauge, $\mathbf{k}=\mathbf{p}-\mathbf{q} ; \gamma_{\mu}$ and $u(p)$ are the Dirac matrices and spinors, while $\psi_{d}(P)$ is the diquark wave function [13]. The factor $1 / 2$ in the quark-quark interaction accounts for the difference of the colour factor compared to the quark-antiquark case.

The effective long-range vector vertex of the quark is defined by [17]

$$
\Gamma_{\mu}(\mathbf{k})=\gamma_{\mu}+\frac{i \kappa}{2 m} \sigma_{\mu \nu} \tilde{k}^{\nu}, \quad \tilde{k}=(0, \mathbf{k}),
$$

where $\kappa$ is the anomalous chromomagnetic moment of quarks.

In the nonrelativistic limit the vector and scalar confining potentials reduce to

$$
\begin{aligned}
V_{\text {conf }}^{V}(r) & =(1-\varepsilon)(A r+B), \\
V_{\text {conf }}^{S}(r) & =\varepsilon(A r+B),
\end{aligned}
$$

where $\varepsilon$ is the mixing coefficient. Thus in this limit the usual Cornell-like potential is reproduced

$$
V(r)=-\frac{4}{3} \frac{\alpha_{s}}{r}+A r+B
$$


TABLE I: Masses $M$ and form factor parameters of diquarks.

\begin{tabular}{clcccc}
\hline \hline $\begin{array}{c}\text { Quark } \\
\text { content }\end{array}$ & $\mathrm{I}$ & $\begin{array}{c}\text { State } \\
n l_{j}\end{array}$ & $\begin{array}{c}M \\
(\mathrm{MeV})\end{array}$ & $\begin{array}{c}\xi \\
(\mathrm{GeV})\end{array}$ & $\begin{array}{c}\zeta \\
\left(\mathrm{GeV}^{2}\right)\end{array}$ \\
\hline$u d$ & 0 & $1 s_{0}$ & 710 & 1.09 & 0.185 \\
& $1 s_{1}$ & 909 & 1.185 & 0.365 \\
& 1 & $1 p_{0}$ & 1.395 & 0.148 \\
& 0 & $1 p_{1}$ & 1397 & 1.452 & 0.195 \\
& 0 & $1 p_{2}$ & 1475 & 1.595 & 0.173 \\
& 0 & $1 p_{1}$ & 1392 & 1.451 & 0.194 \\
& $2 s_{0}$ & 1513 & 1.01 & 0.055 \\
& 1 & 1630 & 1.05 & 0.151 \\
\hline \multirow{2}{*}{$s s$} & 0 & $1 s_{1}$ & 1203 & 1.13 & 0.280 \\
& 0 & $1 s_{1}$ & 1608 & 1.03 & 0.208 \\
& 0 & $2 s_{1}$ & 1817 & 0.805 & 0.235 \\
\hline \hline
\end{tabular}

where we use the QCD coupling constant with freezing

$$
\alpha_{s}\left(\mu^{2}\right)=\frac{4 \pi}{\beta_{0} \ln \frac{\mu^{2}+M_{B}^{2}}{\Lambda^{2}}}, \quad \beta_{0}=11-\frac{2}{3} n_{f}, \quad \mu=\frac{2 m_{1} m_{2}}{m_{1}+m_{2}},
$$

with the background mass $M_{B}=2.24 \sqrt{A}=0.95 \mathrm{GeV}$ [18] and $\Lambda=413 \mathrm{MeV}$ [19].

All parameters of the model were fixed previously from calculations of meson and baryon properties [17]. The constituent quark masses $m_{u}=m_{d}=0.33 \mathrm{GeV}, m_{s}=0.5 \mathrm{GeV}$ and the parameters of the linear potential $A=0.18 \mathrm{GeV}^{2}$ and $B=-0.3 \mathrm{GeV}$ have the usual values of quark models. The value of the mixing coefficient of vector and scalar confining potentials $\varepsilon=-1$ and the universal Pauli interaction constant $\kappa=-1$. Note that the long-range chromomagnetic contribution to the potential, which is proportional to $(1+\kappa)$, vanishes for the chosen value of $\kappa=-1$.

First we calculate masses and form factors of the diquarks. The quasipotential equation (1) is solved numerically for the complete relativistic potential which depends on the diquark mass in a complicated highly nonlinear way [13]. In our approach we assume that diquarks in strange baryons are formed by the constituent quarks of the same mass, i.e. we consider only the $u d, u u, d d$ and $s s$ diquarks. Note that the ground state $u d$ diquark can be both in scalar and axial vector state, while the ground state diquarks composed from quarks of the same flavour $u u, d d$ and $s s$ can be only in the axial vector state due to the Pauli principle. The obtained masses of the ground and excited states of diquarks are presented in Table \. The diquark state is characterized by the quark content, isospin $I$, radial quantum number $n=1,2,3 \ldots$, orbital momentum $l=s, p$ and total angular momentum $j=0,1,2$ (the diquark spin). In this table we also give the values of the parameters $\xi$ and $\zeta$. They enter the vertex $\left\langle d(P)\left|J_{\mu}\right| d(Q)\right\rangle$ of the diquark-gluon interaction (5) which is parameterized by the form factor

$$
F(r)=1-e^{-\xi r-\zeta r^{2}},
$$

that takes the internal diquark structure into account [13]. 
Next we calculate the masses of heavy baryons as the bound states of a quark and diquark. The quark-diquark potential is the sum of spin-independent and spin-dependent parts [13, 14]

$$
V(r)=V_{\mathrm{SI}}(r)+V_{\mathrm{SD}}(r) .
$$

The spin-independent $V_{\mathrm{SI}}(r)$ part is given by

$$
\begin{aligned}
V_{\mathrm{SI}}(r)= & \hat{V}_{\text {Coul }}(r)+V_{\text {conf }}(r)+\frac{1}{E_{d} E_{q}}\left\{\frac{1}{2}\left(E_{q}^{2}-m_{q}^{2}+E_{d}^{2}-M_{d}^{2}\right)\left[\hat{V}_{\text {Coul }}(r)+V_{\text {conf }}^{V}(r)\right]\right. \\
& \left.+\frac{1}{4} \Delta\left[2 V_{\text {Coul }}(r)+V_{\text {conf }}^{V}(r)\right]+\hat{V}_{\text {Coul }}^{\prime}(r) \frac{\mathbf{L}^{2}}{2 r}\right\}+\frac{1}{E_{q}\left(E_{q}+m_{q}\right)}\left\{-\left(E_{q}^{2}-m_{q}^{2}\right) V_{\text {conf }}^{S}(r)\right. \\
& \left.+\frac{1}{4} \Delta\left(\hat{V}_{\text {Coul }}(r)-V_{\text {conf }}(r)-2\left[\frac{E_{q}-m_{q}}{2 m_{q}}-(1+\kappa) \frac{E_{q}+m_{q}}{2 m_{q}}\right] V_{\text {conf }}^{V}(r)\right)\right\},
\end{aligned}
$$

where the diquark and quark energies are defined by their on-mass-shell values [13]

$$
E_{d}=\frac{M^{2}-m_{q}^{2}+M_{d}^{2}}{2 M}, \quad E_{q}=\frac{M^{2}-M_{d}^{2}+m_{q}^{2}}{2 M} .
$$

Here $\Delta$ is the Laplace operator, and $\hat{V}_{\text {Coul }}(r)$ is the smeared Coulomb potential which accounts for the diquark internal structure

$$
\hat{V}_{\text {Coul }}(r)=-\frac{4}{3} \alpha_{s} \frac{F(r)}{r} .
$$

The spin-dependent potential has the following form [14]

$$
V_{\mathrm{SD}}(r)=a_{1} \mathbf{L S}_{d}+a_{2} \mathbf{L S}_{q}+b\left[-\mathbf{S}_{d} \mathbf{S}_{q}+\frac{3}{r^{2}}\left(\mathbf{S}_{d} \mathbf{r}\right)\left(\mathbf{S}_{q} \mathbf{r}\right)\right]+c \mathbf{S}_{d} \mathbf{S}_{q},
$$

where $\mathbf{L}$ is the orbital angular momentum; $\mathbf{S}_{d}$ and $\mathbf{S}_{q}$ are the diquark and quark spin operators, respectively. The first two terms are the spin-orbit interactions, the third one is the tensor interaction and the last one is the spin-spin interaction. The coefficients $a_{1}, a_{2}$, $b$ and $c$ are expressed through the corresponding derivatives of the smeared Coulomb and confining potentials:

$$
\begin{aligned}
a_{1}= & \frac{1}{M_{d}\left(E_{d}+M_{d}\right)} \frac{1}{r}\left[\frac{M_{d}}{E_{d}} \hat{V}_{\text {Coul }}^{\prime}(r)-V_{\text {conf }}^{\prime}(r)\right] \\
& +\frac{1}{E_{d} E_{q}} \frac{1}{r}\left[\hat{V}_{\text {Coul }}^{\prime}(r)+\frac{E_{d}}{M_{d}}\left(\frac{E_{d}-M_{d}}{E_{q}+m_{q}}+\frac{E_{q}-m_{q}}{E_{d}+M_{d}}\right) V_{\text {conf }}^{\prime S}(r)\right], \\
a_{2}= & \frac{1}{E_{d} E_{q}} \frac{1}{r}\left\{\hat{V}_{\text {Coul }}^{\prime}(r)-\left[\frac{E_{q}-m_{q}}{2 m_{q}}-(1+\kappa) \frac{E_{q}+m_{q}}{2 m_{q}}\right] V_{\text {conf }}^{\prime V}(r)\right\} \\
& +\frac{1}{E_{q}\left(E_{q}+m_{q}\right)} \frac{1}{r}\left\{\hat{V}_{\text {Coul }}^{\prime}(r)-V_{\text {conf }}^{\prime}(r)-2\left[\frac{E_{q}-m_{q}}{2 m_{q}}-(1+\kappa) \frac{E_{q}+m_{q}}{2 m_{q}}\right] V_{\text {conf }}^{\prime V}(r)\right\}, \\
b= & \frac{1}{3} \frac{1}{E_{d} E_{q}}\left\{\frac{1}{r} \hat{V}_{\text {Coul }}^{\prime}(r)-\hat{V}_{\text {Coul }}^{\prime \prime}(r)\right\},
\end{aligned}
$$




$$
c=\frac{2}{3} \frac{1}{E_{d} E_{q}} \Delta \hat{V}_{\text {Coul }}(r)
$$

Note that both the one-gluon exchange and confining potentials contribute to the quarkdiquark spin-orbit interaction. The presence of the spin-orbit $\mathbf{L S}_{q}$ and of the tensor terms in the quark-diquark potential (14)-(16) leads to a mixing of states with the same total angular momentum $J$ and parity $P$ but different diquark angular momentum $\left(\mathbf{L}+\mathbf{S}_{d}\right)$. We consider such mixing in the same way as in the case of doubly heavy baryons [12].

\section{STRANGE BARYON MASSES}

We solve numerically the quasipotential equation with the nonperturbative account for the relativistic dynamics both of quarks and diquarks. The calculated values of the ground and excited state baryon masses are presented in Tables II VIII in comparison with available experimental data [1]. In the first column we show the baryon total spin $J$ and parity $P$. In the next three columns experimental candidates are listed with their status and measured mass. In the fifth column we give the states of the quark-diquark system in a baryon and the quark-quark state in a diquark for which the following notations are used: $N L n l_{j}$, where we first show the radial quantum number in the quark-diquark bound system $(N=1,2,3 \ldots)$ and its orbital momentum by a capital letter $(L=S, P, D \ldots)$, then the radial quantum number of two quarks in a diquark $(n=1,2,3 \ldots)$, their orbital momentum by a lowercase letter $(l=s, p, d \ldots)$ and their total momentum $j$ (the diquark spin) in the subscript. Finally, in the last column our predictions for baryon masses are presented.

From Tables IIVIII we see that most of the observed 3- and 4-star states of strange baryons can be well described as ground and excited states of the quark-diquark bound system in which diquark is in the ground either scalar or axial vector state. However not all of these experimental states can be reproduced. Main deviations from this picture are found in the $\Lambda$ sector which is better studied experimentally. Indeed the observed $\frac{1}{2}^{-} 4$-star states $\Lambda(1405)$ and $\Lambda(1670) ; \frac{3}{2}^{-}$4-star states $\Lambda(1520)$ and $\Lambda(1690) ; \frac{1}{2}^{+} 3$-star states $\Lambda(1600)$ and $\Lambda(1810)$ as well as $\frac{5}{2}^{+} 4$-star $\Lambda(1820)$ and 3 -star $\Lambda(2110)$ cannot be simultaneously described in such a simple picture since their mass differences (about $200 \mathrm{MeV}$ ) are too small to be attributed to the radial excitations in the quark-diquark bound system amounting to about $500 \mathrm{MeV}$. Therefore the consideration of excitations inside diquarks is necessary. As we can see from Tables IIVIII the account of diquark excitations allows us to describe all these states and, as a result, to get good agreement of the obtained predictions with data.

In Tables IX XII we compare the results of our model with previous predictions in various theoretical approaches. The strange baryons were treated in a relativized version of the quark potential model in Ref. [20]. The relativistically covariant quark model based on the Bethe-Salpeter equation with instantaneous two- and three-body forces was employed in Ref. [21]. In Ref. [22] the relativistic quark model with the interquark interaction arising from the meson exchange was used. The authors of Ref. [23] made their calculations of baryon masses below $2 \mathrm{GeV}$ in the relativistic interacting quark-diquark model with the Gürsey and Radicati-inspired exchange interaction. Note that in contrast to our approach, all possible types of ground-state scalar and axial vector diquarks, including $q s(q=u$ or $d)$, were used in Ref. [23], but excitations of diquarks were not considered. Finally, the results of lattice calculations with two light dynamical chirally improved quarks corresponding to pion masses between 255 and $596 \mathrm{MeV}$ [24] are given. 
TABLE II: Masses of the positive-parity $\Lambda$ states (in MeV).

\begin{tabular}{|c|c|c|c|c|c|}
\hline \multirow[b]{2}{*}{$J^{P}$} & \multicolumn{3}{|c|}{ Experiment [1] } & \multicolumn{2}{|c|}{ Theory } \\
\hline & State & Status & Mass & $\overline{N L n l_{j}}$ & Mass \\
\hline \multirow{15}{*}{$\frac{1}{2}^{+}$} & $\Lambda$ & $* * * *$ & $1115.683 \pm 0.006$ & $1 S 1 s_{0}$ & 1115 \\
\hline & $\Lambda(1600)$ & $* * *$ & $1560-1600$ & $2 S 1 s_{0}$ & 1615 \\
\hline & $\Lambda(1710)$ & $*$ & $1713 \pm 13$ & & \\
\hline & $\Lambda(1810)$ & $* * *$ & $1750-1810$ & $1 P 1 p_{1}$ & 1901 \\
\hline & & & & $1 S 2 s_{0}$ & 1972 \\
\hline & & & & $1 P 1 p_{0}$ & 1986 \\
\hline & & & & $1 P 1 p_{2}$ & 2042 \\
\hline & & & & $3 S 1 s_{0}$ & 2099 \\
\hline & & & & $1 P 1 p_{1}$ & 2205 \\
\hline & & & & $2 P 1 p_{0}$ & 2431 \\
\hline & & & & $2 S 2 s_{0}$ & 2433 \\
\hline & & & & $4 S 1 s_{0}$ & 2546 \\
\hline & & & & $2 P 1 p_{1}$ & 2559 \\
\hline & & & & $2 P 1 p_{2}$ & 2657 \\
\hline & & & & $2 P 1 p_{1}$ & 2687 \\
\hline \multirow[t]{12}{*}{$\overline{\frac{3}{2}}^{+}$} & $\Lambda(1890)$ & $* * * *$ & $1850-1890$ & $1 D 1 s_{0}$ & 1854 \\
\hline & & & & $1 P 1 p_{2}$ & 1976 \\
\hline & & & & $1 P 1 p_{0}$ & 2130 \\
\hline & & & & $1 P 1 p_{1}$ & 2184 \\
\hline & & & & $1 P 1 p_{2}$ & 2202 \\
\hline & & & & $1 P 1 p_{1}$ & 2212 \\
\hline & & & & $2 D 1 s_{0}$ & 2289 \\
\hline & & & & $2 P 1 p_{0}$ & 2623 \\
\hline & & & & $2 P 1 p_{2}$ & 2629 \\
\hline & & & & $2 P 1 p_{1}$ & 2690 \\
\hline & & & & $2 P 1 p_{1}$ & 2697 \\
\hline & & & & $2 P 1 p_{2}$ & 2701 \\
\hline \multirow[t]{8}{*}{$\frac{5}{2}^{+}$} & $\Lambda(1820)$ & $* * * *$ & $1815-1820$ & $1 D 1 s_{0}$ & 1825 \\
\hline & $\Lambda(2110)$ & $* * *$ & $2090-2110$ & $1 P 1 p_{2}$ & 2098 \\
\hline & & & & $1 P 1 p_{2}$ & 2221 \\
\hline & & & & $1 P 1 p_{1}$ & 2255 \\
\hline & & & & $2 D 1 s_{0}$ & 2258 \\
\hline & & & & $2 P 1 p_{2}$ & 2683 \\
\hline & & & & $2 P 1 p_{2}$ & 2724 \\
\hline & & & & $2 P 1 p_{1}$ & 2746 \\
\hline \multirow[t]{4}{*}{$\overline{7}^{+}$} & $\Lambda(2020)$ & $*$ & $\approx 2020$ & $1 P 1 p_{2}$ & 2251 \\
\hline & & & & $1 G 1 s_{0}$ & 2471 \\
\hline & & & & $1 F 1 p_{0}$ & 2626 \\
\hline & & & & $2 P 1 p_{2}$ & 2744 \\
\hline $\begin{array}{l}\frac{9}{2}^{+} \\
\end{array}$ & $\Lambda(2350)$ & $* * *$ & $2340-2350$ & $1 G 1 s_{0}$ & 2360 \\
\hline
\end{tabular}


TABLE III: Masses of the negative-parity $\Lambda$ states (in $\mathrm{MeV}$ ).

\begin{tabular}{|c|c|c|c|c|c|}
\hline \multirow[b]{2}{*}{$J^{P}$} & \multicolumn{3}{|c|}{ Experiment [1] } & \multicolumn{2}{|c|}{ Theory } \\
\hline & State & Status & Mass & $\overline{N L n l_{j}}$ & Mass \\
\hline \multirow[t]{11}{*}{$\frac{1}{2}^{-}$} & $\Lambda(1405)$ & $* * * *$ & $1405.1_{-1.0}^{+1.3}$ & $1 P 1 s_{0}$ & 1406 \\
\hline & $\Lambda(1670)$ & $* * * *$ & $1660-1670$ & $1 S 1 p_{1}$ & 1667 \\
\hline & $\Lambda(1800)$ & $* * *$ & $1720-1800$ & $1 S 1 p_{0}$ & 1733 \\
\hline & & & & $2 P 1 s_{0}$ & 1927 \\
\hline & & & & $2 S 1 p_{0}$ & 2197 \\
\hline & & & & $1 P 2 s_{0}$ & 2218 \\
\hline & & & & $3 P 1 s_{0}$ & 2274 \\
\hline & & & & $2 S 1 p_{1}$ & 2290 \\
\hline & & & & $1 D 1 p_{1}$ & 2427 \\
\hline & & & & $1 D 1 p_{2}$ & 2491 \\
\hline & & & & $3 S 1 p_{0}$ & 2707 \\
\hline \multirow[t]{13}{*}{$\frac{3}{2}^{-}$} & $\Lambda(1520)$ & $* * * *$ & $1519.5 \pm 1.0$ & $1 P 1 s_{0}$ & 1549 \\
\hline & $\Lambda(1690)$ & $* * * *$ & $1685-1690$ & $1 S 1 p_{2}$ & 1693 \\
\hline & & & & $1 S 1 p_{1}$ & 1812 \\
\hline & $\Lambda(2050)$ & $*$ & $2056 \pm 22$ & $2 P 1 s_{0}$ & 2035 \\
\hline & & & & $1 P 2 s_{0}$ & 2319 \\
\hline & $\Lambda(2325)$ & $*$ & $\approx 2325$ & $2 S 1 p_{2}$ & 2322 \\
\hline & & & & $2 S 1 p_{1}$ & 2392 \\
\hline & & & & $3 P 1 s_{0}$ & 2454 \\
\hline & & & & $1 D 1 p_{0}$ & 2468 \\
\hline & & & & $1 D 1 p_{1}$ & 2523 \\
\hline & & & & $1 D 1 p_{1}$ & 2546 \\
\hline & & & & $1 D 1 p_{2}$ & 2594 \\
\hline & & & & $1 D 1 p_{2}$ & 2622 \\
\hline \multirow[t]{8}{*}{$\frac{5}{2}^{-}$} & $\Lambda(1830)$ & $* * * *$ & $1810-1830$ & $1 S 1 p_{2}$ & 1861 \\
\hline & & & & $1 F 1 s_{0}$ & 2136 \\
\hline & & & & $1 D 1 p_{0}$ & 2350 \\
\hline & & & & $2 S 1 p_{2}$ & 2441 \\
\hline & & & & $1 D 1 p_{1}$ & 2549 \\
\hline & & & & $1 D 1 p_{1}$ & 2560 \\
\hline & & & & $1 D 1 p_{2}$ & 2625 \\
\hline & & & & $1 D 1 p_{2}$ & 2639 \\
\hline \multirow[t]{4}{*}{$\frac{7}{2}^{-}$} & $\Lambda(2100)$ & $* * * *$ & $2090-2100$ & $1 F 1 s_{0}$ & 2097 \\
\hline & & & & $1 D 1 p_{1}$ & 2583 \\
\hline & & & & $1 D 1 p_{2}$ & 2625 \\
\hline & & & & $1 D 1 p_{2}$ & 2639 \\
\hline \multirow[t]{2}{*}{$\frac{9}{2}$} & & & & $1 D 1 p_{2}$ & 2665 \\
\hline & & & & $1 H 1 s_{0}$ & 2738 \\
\hline$\frac{11}{2}$ & & & & $1 H 1 s_{0}$ & 2605 \\
\hline
\end{tabular}


TABLE IV: Masses of the positive-parity $\Sigma$ states (in MeV).

\begin{tabular}{|c|c|c|c|c|c|}
\hline \multirow[b]{2}{*}{$J^{P}$} & \multicolumn{3}{|c|}{ Experiment [1] } & \multicolumn{2}{|c|}{ Theory } \\
\hline & State & Status & Mass & $\overline{N L n l_{j}}$ & Mass \\
\hline \multirow[t]{13}{*}{$\frac{1}{2}^{+}$} & $\Sigma$ & $* * * *$ & $1189.37 \pm 0.07$ & $1 S 1 s_{1}$ & 1187 \\
\hline & $\Sigma(1660)$ & $* * *$ & $1630-1660$ & $2 S 1 s_{1}$ & 1711 \\
\hline & $\Sigma(1770)$ & $*$ & $\approx 1770$ & $1 P 1 p_{1}$ & 1922 \\
\hline & $\Sigma(1880)$ & $*$ & $\approx 1880$ & $1 D 1 s_{1}$ & 1983 \\
\hline & & & & $1 S 2 s_{1}$ & 2028 \\
\hline & & & & $1 P 1 p_{1}$ & 2180 \\
\hline & & & & $3 S 1 s_{1}$ & 2292 \\
\hline & & & & $2 D 1 s_{1}$ & 2472 \\
\hline & & & & $2 P 1 p_{1}$ & 2515 \\
\hline & & & & $2 S 2 s_{1}$ & 2530 \\
\hline & & & & $2 P 1 p_{1}$ & 2647 \\
\hline & & & & $1 D 2 s_{1}$ & 2672 \\
\hline & & & & $4 S 1 s_{1}$ & 2740 \\
\hline \multirow[t]{14}{*}{$\frac{3}{2}^{+}$} & $\Sigma(1385)$ & **** & $1382.80 \pm 0.35$ & $1 S 1 s_{1}$ & 1381 \\
\hline & $\Sigma(1730)$ & $*$ & $1727 \pm 27$ & & \\
\hline & $\Sigma(1840)$ & $*$ & $\approx 1840$ & $2 S 1 s_{1}$ & 1862 \\
\hline & $\Sigma(1940)$ & $*$ & $1941 \pm 18$ & $1 D 1 s_{1}$ & 2025 \\
\hline & $\Sigma(2080)$ & $* *$ & $\approx 2080$ & $1 D 1 s_{1}$ & 2076 \\
\hline & & & & $1 S 2 s_{1}$ & 2096 \\
\hline & & & & $1 P 1 p_{1}$ & 2157 \\
\hline & & & & $1 P 1 p_{1}$ & 2186 \\
\hline & & & & $3 S 1 s_{1}$ & 2347 \\
\hline & & & & $2 D 1 s_{1}$ & 2465 \\
\hline & & & & $2 D 1 s_{1}$ & 2483 \\
\hline & & & & $2 S 2 s_{1}$ & 2584 \\
\hline & & & & $2 P 1 p_{1}$ & 2640 \\
\hline & & & & $2 P 1 p_{1}$ & 2654 \\
\hline \multirow[t]{6}{*}{$\frac{5}{2}^{+}$} & $\Sigma(1915)$ & $* * * *$ & $1900-1915$ & $1 D 1 s_{1}$ & 1991 \\
\hline & $\Sigma(2070)$ & $*$ & $\approx 2070$ & $1 D 1 s_{1}$ & 2062 \\
\hline & & & & $1 P 1 p_{1}$ & 2221 \\
\hline & & & & $2 D 1 s_{1}$ & 2459 \\
\hline & & & & $2 D 1 s_{1}$ & 2485 \\
\hline & & & & $2 P 1 p_{1}$ & 2701 \\
\hline \multirow[t]{3}{*}{$\frac{7}{2}^{+}$} & $\Sigma(2030)$ & $* * * *$ & $2025-2030$ & $1 D 1 s_{1}$ & 2033 \\
\hline & & & & $2 D 1 s_{1}$ & 2470 \\
\hline & & & & $1 G 1 s_{1}$ & 2619 \\
\hline \multirow[t]{2}{*}{$\frac{9}{2}^{+}$} & & & & $1 G 1 s_{1}$ & 2548 \\
\hline & & & & $1 G 1 s_{1}$ & 2619 \\
\hline$\frac{11}{2}^{+}$ & & & & $1 G 1 s_{1}$ & 2529 \\
\hline
\end{tabular}


TABLE V: Masses of the negative-parity $\Sigma$ states (in MeV).

\begin{tabular}{|c|c|c|c|c|c|}
\hline \multirow[b]{2}{*}{$J^{P}$} & \multicolumn{3}{|c|}{ Experiment [1] } & \multicolumn{2}{|c|}{ Theory } \\
\hline & State & Status & Mass & $\overline{N L n l_{j}}$ & Mass \\
\hline \multirow{12}{*}{$\frac{1}{2}^{-}$} & $\Sigma(1620)$ & * & $\approx 1620$ & $1 P 1 s_{1}$ & 1620 \\
\hline & & & & $1 S 1 p_{1}$ & 1693 \\
\hline & $\Sigma(1750)$ & $* * *$ & $1730-1750$ & $1 P 1 s_{1}$ & 1747 \\
\hline & $\Sigma(1900)$ & $*$ & $1900 \pm 21$ & $2 P 1 s_{1}$ & 2115 \\
\hline & $\Sigma(2000)$ & $*$ & $\approx 2000$ & $2 P 1 s_{1}$ & 2198 \\
\hline & & & & $2 S 1 p_{1}$ & 2202 \\
\hline & & & & $1 P 2 s_{1}$ & 2289 \\
\hline & & & & $1 D 1 p_{1}$ & 2381 \\
\hline & & & & $1 P 2 s_{1}$ & 2427 \\
\hline & & & & $3 P 1 s_{1}$ & 2630 \\
\hline & & & & $3 P 1 s_{1}$ & 2634 \\
\hline & & & & $3 S 1 p_{1}$ & 2742 \\
\hline \multirow[t]{14}{*}{$\frac{3}{2}^{-}$} & $\Sigma(1580)$ & * & $\approx 1580$ & & \\
\hline & $\Sigma(1670)$ & $* * *$ & $1665-1670$ & $1 P 1 s_{1}$ & 1706 \\
\hline & & & & $1 P 1 s_{1}$ & 1731 \\
\hline & $\Sigma(1940)$ & $* * *$ & $1900-1940$ & $1 S 1 p_{1}$ & 1856 \\
\hline & & & & $2 P 1 s_{1}$ & 2175 \\
\hline & & & & $2 P 1 s_{1}$ & 2203 \\
\hline & & & & $2 S 1 p_{1}$ & 2300 \\
\hline & & & & $1 F 1 s_{1}$ & 2409 \\
\hline & & & & $1 P 2 s_{1}$ & 2410 \\
\hline & & & & $1 P 2 s_{1}$ & 2430 \\
\hline & & & & $1 D 1 p_{1}$ & 2494 \\
\hline & & & & $1 D 1 p_{1}$ & 2513 \\
\hline & & & & $3 P 1 s_{1}$ & 2623 \\
\hline & & & & $3 P 1 s_{1}$ & 2637 \\
\hline \multirow[t]{8}{*}{$\frac{5}{2}$} & $\Sigma(1775)$ & $* * * *$ & $1770-1775$ & $1 P 1 s_{1}$ & 1757 \\
\hline & & & & $2 P 1 s_{1}$ & 2214 \\
\hline & & & & $1 F 1 s_{1}$ & 2347 \\
\hline & & & & $1 P 2 s_{1}$ & 2459 \\
\hline & & & & $1 F 1 s_{1}$ & 2475 \\
\hline & & & & $1 D 1 p_{1}$ & 2516 \\
\hline & & & & $1 D 1 p_{1}$ & 2524 \\
\hline & & & & $3 P 1 s_{1}$ & 2644 \\
\hline \multirow[t]{3}{*}{$\frac{7}{2}$} & $\Sigma(2100)$ & * & $\approx 2100$ & $1 F 1 s_{1}$ & 2259 \\
\hline & & & & $1 F 1 s_{1}$ & 2349 \\
\hline & & & & $1 D 1 p_{1}$ & 2545 \\
\hline$\frac{9}{2}$ & & & & $1 F 1 s_{1}$ & 2289 \\
\hline
\end{tabular}


TABLE VI: Masses of the positive-parity $\Xi$ states (in MeV).

\begin{tabular}{|c|c|c|c|c|c|}
\hline \multirow[b]{2}{*}{$J^{P}$} & \multicolumn{3}{|c|}{ Experiment [1] } & \multicolumn{2}{|c|}{ Theory } \\
\hline & State & Status & Mass & $\overline{N L n l_{j}}$ & Mass \\
\hline \multirow[t]{12}{*}{$\overline{\frac{1}{2}}^{+}$} & $\Xi$ & $* * * *$ & $1321.71 \pm 0.07$ & $1 S 1 s_{1}$ & 1330 \\
\hline & & & & $2 S 1 s_{1}$ & 1886 \\
\hline & & & & $1 D 1 s_{1}$ & 1993 \\
\hline & & & & $1 P 1 p_{1}$ & 2012 \\
\hline & & & & $1 S 2 s_{1}$ & 2091 \\
\hline & & & & $1 P 1 p_{1}$ & 2142 \\
\hline & & & & $3 S 1 s_{1}$ & 2367 \\
\hline & & & & $2 S 2 s_{1}$ & 2456 \\
\hline & & & & $2 D 1 s_{1}$ & 2510 \\
\hline & & & & $1 D 2 s_{1}$ & 2565 \\
\hline & & & & $2 P 1 p_{1}$ & 2598 \\
\hline & & & & $2 P 1 p_{1}$ & 2624 \\
\hline \multirow[t]{13}{*}{$\frac{3}{2}^{+}$} & $\Xi(1530)$ & **** & $1531.80 \pm 0.32$ & $1 S 1 s_{1}$ & 1518 \\
\hline & & & & $2 S 1 s_{1}$ & 1966 \\
\hline & & & & $1 D 1 s_{1}$ & 2100 \\
\hline & & & & $1 S 2 s_{1}$ & 2121 \\
\hline & & & & $1 D 1 s_{1}$ & 2122 \\
\hline & & & & $1 P 1 p_{1}$ & 2144 \\
\hline & & & & $1 P 1 p_{1}$ & 2149 \\
\hline & & & & $3 S 1 s_{1}$ & 2421 \\
\hline & & & & $2 S 2 s_{1}$ & 2491 \\
\hline & & & & $2 D 1 s_{1}$ & 2597 \\
\hline & & & & $2 P 1 p_{1}$ & 2640 \\
\hline & & & & $2 D 1 s_{1}$ & 2663 \\
\hline & & & & $2 P 1 p_{1}$ & 2664 \\
\hline \multirow[t]{5}{*}{$\overline{\frac{5}{2}}^{+}$} & & & & $1 D 1 s_{1}$ & 2108 \\
\hline & & & & $1 D 1 s_{1}$ & 2147 \\
\hline & & & & $1 P 1 p_{1}$ & 2213 \\
\hline & & & & $2 D 1 s_{1}$ & 2605 \\
\hline & & & & $2 D 1 s_{1}$ & 2630 \\
\hline \multirow[t]{2}{*}{$\overline{7}^{+}$} & & & & $1 D 1 s_{1}$ & 2189 \\
\hline & & & & $2 D 1 s_{1}$ & 2686 \\
\hline
\end{tabular}

From these tables we see that our diquark model predicts appreciably less states than the three-quark approaches. The differences become apparent with the growth of the orbital and radial excitations in the baryon. Our results turn out to be competitive with their predictions for the masses of the well established (4- and 3-star) resonances, which agree well with experimental data. For the less established (1- and 2-star) states situation is more complicated. 
TABLE VII: Masses of the negative-parity $\Xi$ states (in MeV).

\begin{tabular}{|c|c|c|c|c|c|}
\hline \multirow[b]{2}{*}{$J^{P}$} & \multicolumn{3}{|c|}{ Experiment [1] } & \multicolumn{2}{|c|}{ Theory } \\
\hline & State & Status & Mass & $\mathrm{NLnl}_{j}$ & Mass \\
\hline \multirow[t]{9}{*}{$\frac{1}{2}^{-}$} & & & & $1 P 1 s_{1}$ & 1682 \\
\hline & & & & $1 P 1 s_{1}$ & 1758 \\
\hline & & & & $1 S 1 p_{1}$ & 1839 \\
\hline & & & & $2 P 1 s_{1}$ & 2160 \\
\hline & & & & $2 S 1 p_{1}$ & 2210 \\
\hline & & & & $2 P 1 s_{1}$ & 2233 \\
\hline & & & & $1 P 2 s_{1}$ & 2261 \\
\hline & & & & $1 D 1 p_{1}$ & 2346 \\
\hline & & & & $1 P 2 s_{1}$ & 2347 \\
\hline \multirow[t]{10}{*}{$\overline{\frac{3}{2}}$} & & & & $1 P 1 s_{1}$ & 1764 \\
\hline & $\Xi(1820)$ & $* * *$ & $1823 \pm 5$ & $1 P 1 s_{1}$ & 1798 \\
\hline & & & & $1 S 1 p_{1}$ & 1904 \\
\hline & & & & $2 P 1 s_{1}$ & 2245 \\
\hline & & & & $2 P 1 s_{1}$ & 2252 \\
\hline & & & & $1 P 2 s_{1}$ & 2350 \\
\hline & & & & $1 P 2 s_{1}$ & 2352 \\
\hline & & & & $1 F 1 s_{1}$ & 2400 \\
\hline & & & & $1 D 1 p_{1}$ & 2482 \\
\hline & & & & $1 D 1 p_{1}$ & 2506 \\
\hline \multirow[t]{7}{*}{$\frac{5}{2}$} & & & & $1 P 1 s_{1}$ & 1853 \\
\hline & & & & $2 P 1 s_{1}$ & 2333 \\
\hline & & & & $1 P 2 s_{1}$ & 2411 \\
\hline & & & & $1 F 1 s_{1}$ & 2455 \\
\hline & & & & $1 D 1 p_{1}$ & 2489 \\
\hline & & & & $1 D 1 p_{1}$ & 2545 \\
\hline & & & & $1 F 1 s_{1}$ & 2569 \\
\hline \multirow[t]{3}{*}{$\frac{7}{2}$} & & & & $1 F 1 s_{1}$ & 2460 \\
\hline & & & & $1 F 1 s_{1}$ & 2474 \\
\hline & & & & $1 D 1 p_{1}$ & 2611 \\
\hline$\frac{9}{2}^{-}$ & & & & $1 F 1 s_{1}$ & 2502 \\
\hline
\end{tabular}

First we discuss results for the $\Lambda$ sector. It is necessary to emphasis that the experimental mass of the $\frac{1}{2}^{-} 4$-star $\Lambda(1405)$ is naturally reproduced if this state is considered as the first orbital excitation $1 P$ in the strange quark-light scalar $\left(1 s_{0}\right)$ diquark picture of $\Lambda$ baryons. The rather low mass of this state represents difficulties for most of the three-quark models [20 22], which predict its mass about $100 \mathrm{MeV}$ higher than experimental value. There are no theoretical candidates for the $\frac{1}{2}^{+} 1$-star $\Lambda(1710)$ state. The mass of the $\frac{7}{2}^{+} 1$-star $\Lambda(2020)$ state is predicted somewhat heavier by all models. Other 1-star $\Lambda$ states are well described.

In the $\Sigma$ sector all considered approaches cannot accommodate the $\frac{3}{2}^{-}$1-star $\Sigma(1580)$ 
TABLE VIII: Masses of the $\Omega$ states (in MeV).

\begin{tabular}{|c|c|c|c|c|c|c|c|c|}
\hline \multirow[b]{2}{*}{$J^{P}$} & \multicolumn{3}{|c|}{ Experiment [1] } & \multicolumn{2}{|c|}{ Theory } & \multirow[b]{2}{*}{$J^{P}$} & \multicolumn{2}{|c|}{ Theory } \\
\hline & $\overline{\text { State }}$ & Status & Mass & $\overline{N L n l_{j}}$ & Mass & & $\overline{N L n l_{j}}$ & Mass \\
\hline \multirow[t]{3}{*}{$\frac{1}{2}^{+}$} & & & & $1 D 1 s_{1}$ & 2301 & $\frac{1}{2}$ & $1 P 1 s_{1}$ & 1941 \\
\hline & & & & & & & $2 P 1 s_{1}$ & 2463 \\
\hline & & & & & & & $1 P 2 s_{1}$ & 2580 \\
\hline \multirow[t]{5}{*}{$\frac{3}{2}^{+}$} & $\Omega$ & $* * * *$ & $1672.45 \pm 0.29$ & $1 S 1 s_{1}$ & 1678 & $\frac{3}{2}^{-}$ & $1 P 1 s_{1}$ & 2038 \\
\hline & & & & $2 S 1 s_{1}$ & 2173 & & $2 P 1 s_{1}$ & 2537 \\
\hline & & & & $1 S 2 s_{1}$ & 2304 & & $1 P 2 s_{1}$ & 2636 \\
\hline & & & & $1 D 1 s_{1}$ & 2332 & & & \\
\hline & & & & $3 S 1 s_{1}$ & 2671 & & & \\
\hline$\frac{5}{2}^{+}$ & & & & $1 D 1 s_{1}$ & 2401 & $\frac{5}{2}$ & $1 F 1 s_{1}$ & 2653 \\
\hline \multirow[t]{2}{*}{$\frac{\frac{7}{2}^{+}}{2}$} & & & & $1 D 1 s_{1}$ & 2369 & $\frac{7}{2}$ & $1 F 1 s_{1}$ & 2599 \\
\hline & & & & & & $\frac{9}{2}$ & $1 F 1 s_{1}$ & 2649 \\
\hline
\end{tabular}

state. The predicted lowest mass $\frac{3}{2}^{-}$state corresponds to the 3 -star $\Sigma(1670)$ state. We have no candidate for the $\frac{3}{2}^{+} 1$-star $\Sigma(1730)$ state in our model. The calculated masses of the 1-star $\frac{1}{2}^{+} \Sigma(1770), \frac{1}{2}^{-} \Sigma(1900)$ and $\frac{7}{2}^{-} \Sigma(2100)$ candidates are by more than $100 \mathrm{MeV}$ heavier than experimentally measured masses. All other known 2- and 1-star $\Sigma$ states are described with reasonable accuracy.

In the $\Xi$ sector only three (two 4 - and one 3 -star) states and in the $\Omega$ sector only one (4-star) state of the observed baryons have established quantum numbers. They are well described by our model. We have at least one candidate for each of the other eight $\Xi$ (three of them have 3-stars) and three $\Omega$ (one of them has 3-stars) states given in PDG Listings [1] with the predicted masses close to the experimental values. However it will be too speculative to assign the quantum numbers to these states only on the basis of their masses. More experimental and theoretical input is needed.

\section{REGGE TRAJECTORIES OF STRANGE BARYONS}

In the presented analysis we calculated masses of orbitally excited strange baryons up to rather high orbital excitation numbers: up to $L=5$ in the quark-diquark bound system, where diquark is in the ground state. This makes it possible to construct the strange baryon Regge trajectories:

$$
J=\alpha M^{2}+\alpha_{0},
$$

where $\alpha$ is the slope and $\alpha_{0}$ is the intercept.

In Figs. 1. 3 we plot the Regge trajectories in the $\left(J, M^{2}\right)$ plane for strange baryons with natural $\left(P=(-1)^{J-1 / 2}\right)$ and unnatural $\left(P=(-1)^{J+1 / 2}\right)$ parities. The masses calculated in our model are shown by diamonds. Available experimental data are given by dots with error bars and corresponding baryon names. Straight lines were obtained by the $\chi^{2}$ fit of calculated values. The fitted slopes and intercepts of the Regge trajectories are given in Table XIII. We see that the calculated strange baryon masses lie on the linear trajectories. 
TABLE IX: Comparison of theoretical predictions and experimental data for the masses of the $\Lambda$ states (in $\mathrm{MeV}$ ).

\begin{tabular}{|c|c|c|c|c|c|c|c|c|c|}
\hline \multirow[b]{2}{*}{$J^{P}$} & \multicolumn{3}{|c|}{ Experiment [1] } & \multicolumn{6}{|c|}{ Theory } \\
\hline & State & Status & Mass & Our & [20] & {$[21]$} & {$[22]$} & [23] & [24] \\
\hline \multirow{8}{*}{$\frac{1}{2}^{+}$} & $\Lambda$ & $* * * *$ & $1115.683 \pm 0.006$ & 1115 & 1115 & 1108 & 1136 & 1116 & $1149 \pm 18$ \\
\hline & $\Lambda(1600)$ & $* * *$ & $1560-1600$ & 1615 & 1680 & 1677 & 1625 & 1518 & $1807 \pm 94$ \\
\hline & $\Lambda(1710)$ & $*$ & $1713 \pm 13$ & & & & & & \\
\hline & $\Lambda(1810)$ & $* * *$ & $1750-1810$ & 1901 & 1830 & 1747 & 1799 & 1666 & $2112 \pm 54$ \\
\hline & & & & 1972 & 1910 & 1898 & & 1955 & $2137 \pm 69$ \\
\hline & & & & 1986 & 2010 & 2077 & & 1960 & \\
\hline & & & & 2042 & 2105 & 2099 & & & \\
\hline & & & & 2099 & 2120 & 2132 & & & \\
\hline \multirow[t]{5}{*}{$\frac{3}{2}^{+}$} & $\Lambda(1890)$ & $* * * *$ & $1850-1890$ & 1854 & 1900 & 1823 & & 1896 & $1991 \pm 103$ \\
\hline & & & & 1976 & 1960 & 1952 & & & $2058 \pm 139$ \\
\hline & & & & 2130 & 1995 & 2045 & & & $2481 \pm 111$ \\
\hline & & & & 2184 & 2050 & 2087 & & & \\
\hline & & & & 2202 & 2080 & 2133 & & & \\
\hline \multirow[t]{5}{*}{$\frac{5}{2}^{+}$} & $\Lambda(1820)$ & $* * * *$ & $1815-1820$ & 1825 & 1890 & 1834 & & 1896 & \\
\hline & $\Lambda(2110)$ & $* * *$ & $2090-2110$ & 2098 & 2035 & 1999 & & & \\
\hline & & & & 2221 & 2115 & 2078 & & & \\
\hline & & & & 2255 & 2115 & 2127 & & & \\
\hline & & & & 2258 & 2180 & 2150 & & & \\
\hline \multirow[t]{2}{*}{$\frac{7}{2}^{+}$} & $\Lambda(2020)$ & $*$ & $\approx 2020$ & 2251 & 2120 & 2130 & & & \\
\hline & & & & 2471 & & 2331 & & & \\
\hline$\frac{9}{2}^{+}$ & $\Lambda(2350)$ & $* * *$ & $2340-2350$ & 2360 & & 2340 & & & \\
\hline \multirow[t]{6}{*}{$\frac{1}{2}^{-}$} & $\Lambda(1405)$ & $* * * *$ & $1405.1_{-1.0}^{+1.3}$ & 1406 & 1550 & 1524 & 1556 & 1431 & $1416 \pm 81$ \\
\hline & $\Lambda(1670)$ & $* * * *$ & $1660-1670$ & 1667 & 1615 & 1630 & 1682 & 1443 & $1546 \pm 110$ \\
\hline & $\Lambda(1800)$ & $* * *$ & $1720-1800$ & 1733 & 1675 & 1816 & 1778 & 1650 & $1713 \pm 116$ \\
\hline & & & & 1927 & 2015 & 2011 & & 1732 & $2075 \pm 249$ \\
\hline & & & & 2197 & 2095 & 2076 & & 1785 & \\
\hline & & & & 2218 & 2160 & 2117 & & 1854 & \\
\hline \multirow[t]{9}{*}{$\frac{3}{2}$} & $\Lambda(1520)$ & $* * * *$ & $1519.5 \pm 1.0$ & 1549 & 1545 & 1508 & 1556 & 1431 & $1751 \pm 40$ \\
\hline & $\Lambda(1690)$ & $* * * *$ & $1685-1690$ & 1693 & 1645 & 1662 & 1682 & 1443 & $2203 \pm 106$ \\
\hline & & & & 1812 & 1770 & 1775 & & 1650 & $2381 \pm 87$ \\
\hline & $\Lambda(2050)$ & $*$ & $2056 \pm 22$ & 2035 & 2030 & 1987 & & 1732 & \\
\hline & & & & 2319 & 2110 & 2090 & & 1785 & \\
\hline & $\Lambda(2325)$ & $*$ & $\approx 2325$ & 2322 & 2185 & 2147 & & 1854 & \\
\hline & & & & 2392 & 2230 & 2259 & & 1928 & \\
\hline & & & & 2454 & 2290 & 2275 & & 1969 & \\
\hline & & & & 2468 & & 2313 & & & \\
\hline \multirow[t]{3}{*}{$\frac{5}{2}^{-}$} & $\Lambda(1830)$ & $* * * *$ & $1810-1830$ & 1861 & 1775 & 1828 & 1778 & 1785 & \\
\hline & & & & 2136 & 2180 & 2080 & & & \\
\hline & & & & 2350 & 2250 & 2179 & & & \\
\hline \multirow[t]{2}{*}{$\frac{7}{2}$} & $\Lambda(2100)$ & $* * * *$ & $2090-2100$ & 2097 & 2150 & 2090 & & & \\
\hline & & & & 2583 & 2230 & 2227 & & & \\
\hline$\frac{9}{2}$ & & & & 2665 & & 2370 & & & \\
\hline
\end{tabular}


TABLE X: Comparison of theoretical predictions and experimental data for the masses of the $\Sigma$ states (in $\mathrm{MeV}$ ).

\begin{tabular}{|c|c|c|c|c|c|c|c|c|c|}
\hline \multirow[b]{2}{*}{$J^{P}$} & \multicolumn{3}{|c|}{ Experiment [1] } & \multicolumn{6}{|c|}{ Theory } \\
\hline & State & Status & Mass & Our & {$[20]$} & [21] & {$[22]$} & [23] & [24] \\
\hline \multirow{8}{*}{$\frac{1}{2}^{+}$} & $\Sigma$ & $* * * *$ & $1189.37 \pm 0.07$ & 1187 & 1190 & 1190 & 1180 & 1211 & $1216 \pm 15$ \\
\hline & $\Sigma(1660)$ & $* * *$ & $1630-1660$ & 1711 & 1720 & 1760 & 1616 & 1546 & $2069 \pm 74$ \\
\hline & $\Sigma(1770)$ & $*$ & $\approx 1770$ & 1922 & 1915 & 1947 & 1911 & 1668 & $2149 \pm 66$ \\
\hline & $\Sigma(1880)$ & $*$ & $\approx 1880$ & 1983 & 1970 & 2009 & & 1801 & $2335 \pm 63$ \\
\hline & & & & 2028 & 2005 & 2052 & & & \\
\hline & & & & 2180 & 2030 & 2098 & & & \\
\hline & & & & 2292 & 2105 & 2138 & & & \\
\hline & & & & 2472 & 2195 & & & & \\
\hline \multirow[t]{8}{*}{$\frac{3}{2}^{+}$} & $\Sigma(1385)$ & $* * * *$ & $1382.80 \pm 0.35$ & 1381 & 1370 & 1411 & 1389 & 1334 & $1471 \pm 23$ \\
\hline & $\Sigma(1730)$ & $*$ & $1727 \pm 27$ & & 1920 & 1896 & 1865 & 1439 & \\
\hline & $\Sigma(1840)$ & $*$ & $\approx 1840$ & 1862 & 1970 & 1961 & & 1924 & $2194 \pm 81$ \\
\hline & $\Sigma(1940)$ & $*$ & $1941 \pm 18$ & 2025 & 2010 & 2011 & & & $2250 \pm 79$ \\
\hline & $\Sigma(2080)$ & $* *$ & $\approx 2080$ & 2076 & 2030 & 2044 & & & $2468 \pm 67$ \\
\hline & & & & 2096 & 2045 & 2062 & & & \\
\hline & & & & 2157 & 2085 & 2103 & & & \\
\hline & & & & 2186 & 2115 & 2112 & & & \\
\hline \multirow{3}{*}{$\frac{5}{2}^{+}$} & $\Sigma(1915)$ & $* * * *$ & $1900-1915$ & 1991 & 1995 & 1956 & & 2061 & \\
\hline & $\Sigma(2070)$ & $*$ & $\approx 2070$ & 2062 & 2030 & 2027 & & & \\
\hline & & & & 2221 & 2095 & 2071 & & & \\
\hline \multirow[t]{2}{*}{$\frac{7}{2}^{+}$} & $\Sigma(2030)$ & $* * * *$ & $2025-2030$ & 2033 & 2060 & 2070 & & & \\
\hline & & & & 2470 & 2125 & 2161 & & & \\
\hline \multirow[t]{8}{*}{$\frac{1}{2}$} & $\Sigma(1620)$ & * & $\approx 1620$ & 1620 & 1630 & 1628 & 1677 & 1753 & $1603 \pm 38$ \\
\hline & & & & 1693 & 1675 & 1771 & 1736 & 1868 & $1718 \pm 58$ \\
\hline & $\Sigma(1750)$ & $* * *$ & $1730-1750$ & 1747 & 1695 & 1798 & 1759 & 1895 & $1730 \pm 34$ \\
\hline & $\Sigma(1900)$ & $*$ & $1900 \pm 21$ & 2115 & 2110 & 2111 & & & $2478 \pm 104$ \\
\hline & $\Sigma(2000)$ & $*$ & $\approx 2000$ & 2198 & 2155 & 2136 & & & \\
\hline & & & & 2202 & 2165 & 2251 & & & \\
\hline & & & & 2289 & 2205 & 2264 & & & \\
\hline & & & & 2381 & 2260 & 2288 & & & \\
\hline \multirow[t]{7}{*}{$\frac{3}{2}$} & $\Sigma(1580)$ & * & $\approx 1580$ & & & & & & \\
\hline & $\Sigma(1670)$ & $* * *$ & $1665-1670$ & 1706 & 1655 & 1669 & 1677 & 1753 & $1736 \pm 40$ \\
\hline & & & & 1731 & 1750 & 1728 & 1736 & 1868 & $1861 \pm 20$ \\
\hline & $\Sigma(1940)$ & $* * *$ & $1900-1940$ & 1856 & 1755 & 1781 & 1759 & 1895 & $2297 \pm 122$ \\
\hline & & & & 2175 & 2120 & 2139 & & & $2394 \pm 74$ \\
\hline & & & & 2203 & 2185 & 2171 & & & \\
\hline & & & & 2300 & 2200 & 2203 & & & \\
\hline \multirow[t]{3}{*}{$\frac{5}{2}$} & $\Sigma(1775)$ & $* * * *$ & $1770-1775$ & 1757 & 1755 & 1770 & 1736 & 1753 & \\
\hline & & & & 2214 & 2205 & 2174 & & & \\
\hline & & & & 2347 & 2250 & 2226 & & & \\
\hline \multirow[t]{2}{*}{$\frac{7}{2}$} & $\Sigma(2100)$ & $*$ & $\approx 2100$ & 2259 & 2245 & 2236 & & & \\
\hline & & & & 2349 & & 2285 & & & \\
\hline$\frac{9}{2}$ & & & & 2289 & & 2325 & & & \\
\hline
\end{tabular}


TABLE XI: Comparison of theoretical predictions and experimental data for the masses of the $\Xi$ states (in $\mathrm{MeV}$ ).

\begin{tabular}{|c|c|c|c|c|c|c|c|c|c|}
\hline \multirow[b]{2}{*}{$J^{P}$} & \multicolumn{3}{|c|}{ Experiment [1] } & \multicolumn{6}{|c|}{ Theory } \\
\hline & State & Status & Mass & Our & [20] & [21] & {$[22]$} & {$[23]$} & {$[24]$} \\
\hline \multirow[t]{7}{*}{$\overline{\frac{1}{2}}^{+}$} & $\Xi$ & $* * * *$ & $1321.71 \pm 0.07$ & 1330 & 1305 & 1310 & 1348 & 1317 & $1303 \pm 13$ \\
\hline & & & & 1886 & 1840 & 1876 & 1805 & 1772 & $2178 \pm 48$ \\
\hline & & & & 1993 & 2040 & 2062 & & 1868 & $2231 \pm 44$ \\
\hline & & & & 2012 & 2100 & 2131 & & 1874 & $2408 \pm 45$ \\
\hline & & & & 2091 & 2130 & 2176 & & & \\
\hline & & & & 2142 & 2150 & 2215 & & & \\
\hline & & & & 2367 & 2230 & 2249 & & & \\
\hline \multirow[t]{8}{*}{$\overline{\frac{3}{2}}^{+}$} & $\Xi(1530)$ & $* * * *$ & $1531.80 \pm 0.32$ & 1518 & 1505 & 1539 & 1528 & 1552 & $1553 \pm 18$ \\
\hline & & & & 1966 & 2045 & 1988 & & 1653 & $2228 \pm 44$ \\
\hline & & & & 2100 & 2065 & 2076 & & & $2398 \pm 52$ \\
\hline & & & & 2121 & 2115 & 2128 & & & $2574 \pm 52$ \\
\hline & & & & 2122 & 2165 & 2170 & & & \\
\hline & & & & 2144 & 2170 & 2175 & & & \\
\hline & & & & 2149 & 2210 & 2219 & & & \\
\hline & & & & 2421 & 2230 & 2257 & & & \\
\hline \multirow[t]{3}{*}{$\overline{\frac{5}{2}}^{+}$} & & & & 2108 & 2045 & 2013 & & & \\
\hline & & & & 2147 & 2165 & 2141 & & & \\
\hline & & & & 2213 & 2230 & 2197 & & & \\
\hline$\frac{7}{2}^{+}$ & & & & 2189 & 2180 & 2169 & & & \\
\hline \multirow[t]{7}{*}{$\frac{1}{2}$} & & & & 1682 & 1755 & 1770 & & & $1716 \pm 43$ \\
\hline & & & & 1758 & 1810 & 1922 & & & $1837 \pm 28$ \\
\hline & & & & 1839 & 1835 & 1938 & & & $1844 \pm 43$ \\
\hline & & & & 2160 & 2225 & 2241 & & & $2758 \pm 78$ \\
\hline & & & & 2210 & 2285 & 2266 & & & \\
\hline & & & & 2233 & 2300 & 2387 & & & \\
\hline & & & & 2261 & 2320 & 2411 & & & \\
\hline \multirow[t]{7}{*}{$\frac{3}{2}^{-}$} & & & & 1764 & 1785 & 1780 & 1792 & 1861 & $1894 \pm 38$ \\
\hline & $\Xi(1820)$ & $* * *$ & $1823 \pm 5$ & 1798 & 1880 & 1873 & & 1971 & $1906 \pm 29$ \\
\hline & & & & 1904 & 1895 & 1924 & & & $2426 \pm 73$ \\
\hline & & & & 2245 & 2240 & 2246 & & & $2497 \pm 61$ \\
\hline & & & & 2252 & 2305 & 2284 & & & \\
\hline & & & & 2350 & 2330 & 2353 & & & \\
\hline & & & & 2352 & 2340 & 2384 & & & \\
\hline \multirow[t]{3}{*}{$\frac{5}{2}$} & & & & 1853 & 1900 & 1955 & 1881 & & \\
\hline & & & & 2333 & 2345 & 2292 & & & \\
\hline & & & & 2411 & 2350 & 2409 & & & \\
\hline \multirow[t]{2}{*}{$\overline{\frac{7}{2}}$} & & & & 2460 & 2355 & 2320 & & & \\
\hline & & & & 2474 & & 2425 & & & \\
\hline$\frac{9}{2}^{-}$ & & & & 2502 & & 2505 & & & \\
\hline
\end{tabular}


TABLE XII: Comparison of theoretical predictions and experimental data for the masses of the $\Omega$ states (in $\mathrm{MeV}$ ).

\begin{tabular}{|c|c|c|c|c|c|c|c|c|}
\hline \multirow[b]{2}{*}{$J^{P}$} & \multicolumn{3}{|c|}{ Experiment [1] } & \multicolumn{5}{|c|}{ Theory } \\
\hline & State & Status & Mass & Our & [20] & {$[21]$} & {$[23]$} & {$[24]$} \\
\hline \multirow[t]{2}{*}{$\overline{\frac{1}{2}}^{+}$} & & & & 2301 & 2220 & 2232 & & $2350 \pm 63$ \\
\hline & & & & & 2255 & 2256 & & $2481 \pm 51$ \\
\hline \multirow[t]{4}{*}{$\frac{3}{2}^{+}$} & $\Omega$ & $* * * *$ & $1672.45 \pm 0.29$ & 1678 & 1635 & 1636 & 1672 & $1642 \pm 17$ \\
\hline & & & & 2173 & 2165 & 2177 & & $2470 \pm 49$ \\
\hline & & & & 2304 & 2280 & 2236 & & \\
\hline & & & & 2332 & 2345 & 2287 & & \\
\hline \multirow[t]{2}{*}{$\overline{\frac{5}{2}}^{+}$} & & & & 2401 & 2280 & 2253 & & \\
\hline & & & & & 2345 & 2312 & & \\
\hline$\frac{7}{2}^{+}$ & & & & 2369 & 2295 & 2292 & & \\
\hline \multirow[t]{3}{*}{$\frac{1}{2}$} & & & & 1941 & 1950 & 1992 & & $1944 \pm 56$ \\
\hline & & & & 2463 & 2410 & 2456 & & $2716 \pm 118$ \\
\hline & & & & 2580 & 2490 & 2498 & & \\
\hline \multirow[t]{3}{*}{$\overline{\frac{3}{2}}$} & & & & 2038 & 2000 & 1976 & & $2049 \pm 32$ \\
\hline & & & & 2537 & 2440 & 2446 & & $2755 \pm 67$ \\
\hline & & & & 2636 & 2495 & 2507 & & \\
\hline$\frac{5}{2}$ & & & & 2653 & 2490 & 2528 & & \\
\hline$\frac{7}{2}$ & & & & 2599 & & 2531 & & \\
\hline$\frac{9}{2}$ & & & & 2649 & & 2606 & & \\
\hline
\end{tabular}

TABLE XIII: Fitted parameters $\alpha, \alpha_{0}$ for the slope and intercept of the $\left(J, M^{2}\right)$ Regge trajectories of strange baryons.

\begin{tabular}{lcc||ccc}
\hline \hline Baryon & $\alpha\left(\mathrm{GeV}^{-2}\right)$ & $\alpha_{0}$ & Baryon & $\alpha\left(\mathrm{GeV}^{-2}\right)$ & $\alpha_{0}$ \\
\hline$\Lambda\left(\frac{1}{2}^{+}\right)$ & $0.923 \pm 0.016$ & $-0.648 \pm 0.057$ & $\Lambda\left(\frac{1}{2}^{-}\right)$ & $0.732 \pm 0.018$ & $-0.951 \pm 0.074$ \\
$\Sigma\left(\frac{1}{2}^{+}\right)$ & $0.799 \pm 0.029$ & $-0.676 \pm 0.100$ & $\Sigma\left(\frac{3}{2}^{+}\right)$ & $0.897 \pm 0.010$ & $-0.225 \pm 0.037$ \\
$\Xi\left(\frac{1}{2}^{+}\right)$ & $0.694 \pm 0.007$ & $-0.721 \pm 0.024$ & $\Xi\left(\frac{3}{2}^{+}\right)$ & $0.769 \pm 0.032$ & $-0.249 \pm 0.098$ \\
& & & $\Omega\left(\frac{3}{2}^{+}\right)$ & $0.712 \pm 0.002$ & $-0.504 \pm 0.007$ \\
\hline \hline
\end{tabular}

The natural parity $\Lambda$ Regge trajectory is the best studied experimentally. There are five well established (four 4-star and one 3-star) states [1] on this trajectory. The masses of these states calculated in our model agree well with data. Using the constructed Regge trajectory we can predict the mass of the $\frac{11}{2}^{-} \Lambda$ state to be about $2605 \mathrm{MeV}$ (see Table III). This state could contribute to the $\Lambda(2585)$ bumps observed with the mass $\approx 2585 \mathrm{MeV}$ [1]. Each of the $\Sigma$ Regge trajectories contains three well established states [1], well fitting to the strait lines. Other trajectories are less motivated experimentally and contain at most two well established states.

Using the values of the slopes and intercepts of the Regge trajectories of the $\frac{3}{2}^{+}$strange baryons we can test the validity of the relations between them proposed in the literature 


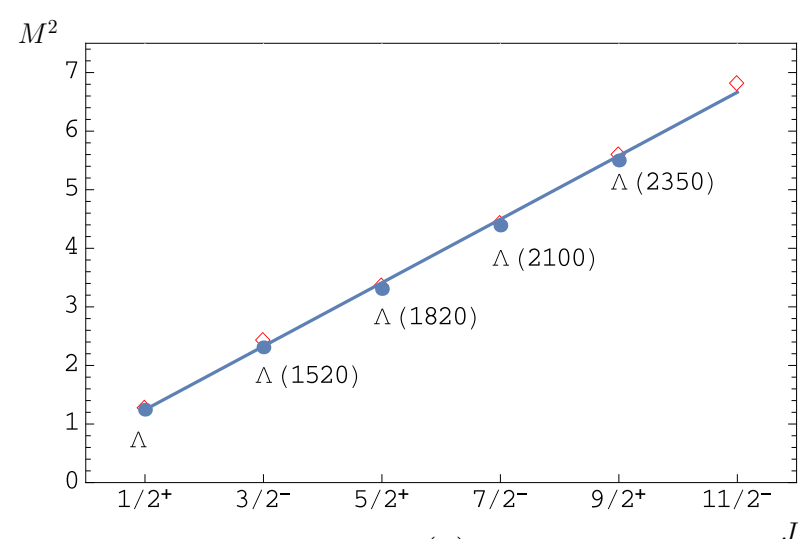

(a)

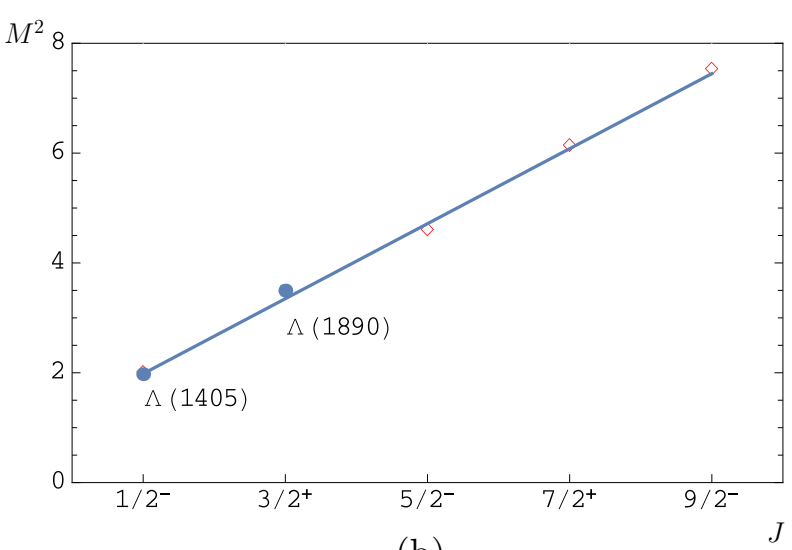

(b)

FIG. 1: The $\left(J, M^{2}\right)$ Regge trajectories for the $\Lambda$ baryons with natural (a) and unnatural (b) parities. Diamonds are predicted masses. Available experimental data are given by dots with particle names; $M^{2}$ is in $\mathrm{GeV}^{2}$.

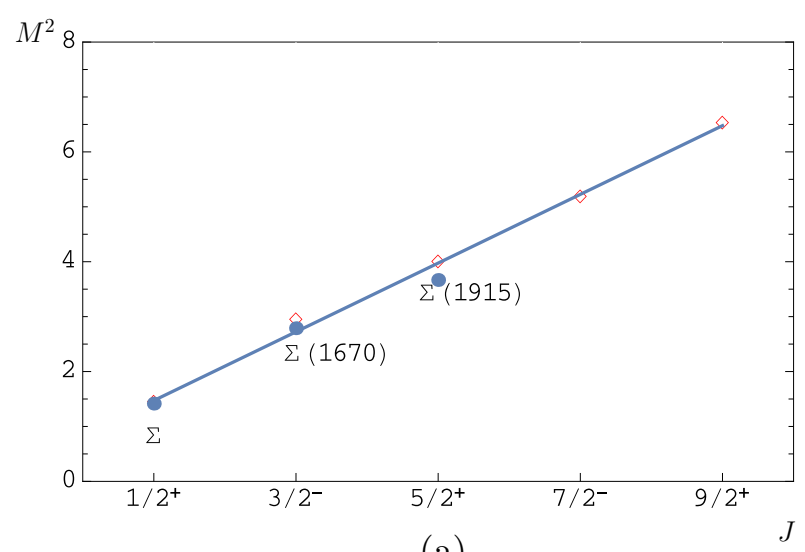

(a)

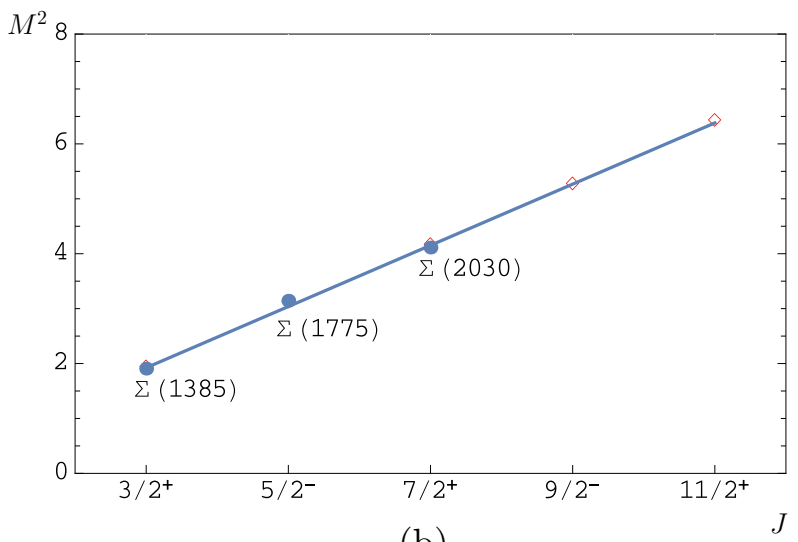

(b)

FIG. 2: Same as in Fig. 1 1 for the $\Sigma$ baryons.

(see e.g. [25-27] and references therein). It is easy to check that the additivity of inverse slopes

$$
\frac{1}{\alpha\left(\Sigma^{*}\right)}+\frac{1}{\alpha(\Omega)}=\frac{2}{\alpha\left(\Xi^{*}\right)}
$$

factorization of slopes

$$
\alpha\left(\Sigma^{*}\right) \alpha(\Omega)=\alpha^{2}\left(\Xi^{*}\right)
$$

and additivity of intercepts

$$
\alpha_{0}\left(\Sigma^{*}\right)+\alpha_{0}(\Omega)=2 \alpha_{0}\left(\Xi^{*}\right)
$$

are well satisfied. Indeed, in the left hand side of Eq. (19) we get $2.52 \pm 0.02$ and in the right hand side $2.60 \pm 0.11$; for Eq. (20) the corresponding values are $0.639 \pm 0.010$ and $0.592 \pm 0.050$, while for Eq. (21) they are $-0.729 \pm 0.044$ and $-0.498 \pm 0.196$.

We can also compare the calculated slopes of the strange baryon Regge trajectories with our previous results for the slopes of heavy baryons [14] and light mesons [19]. Such comparison shows that the strange baryon slopes lie just in between the corresponding slopes 


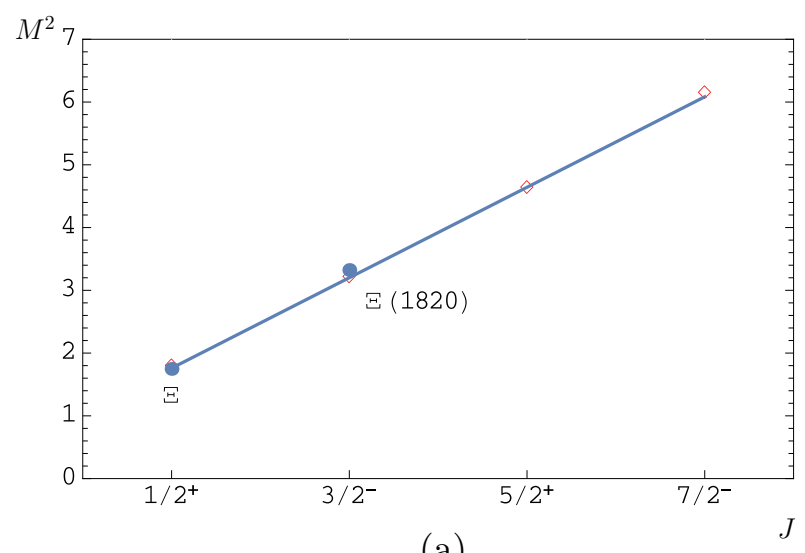

(a)

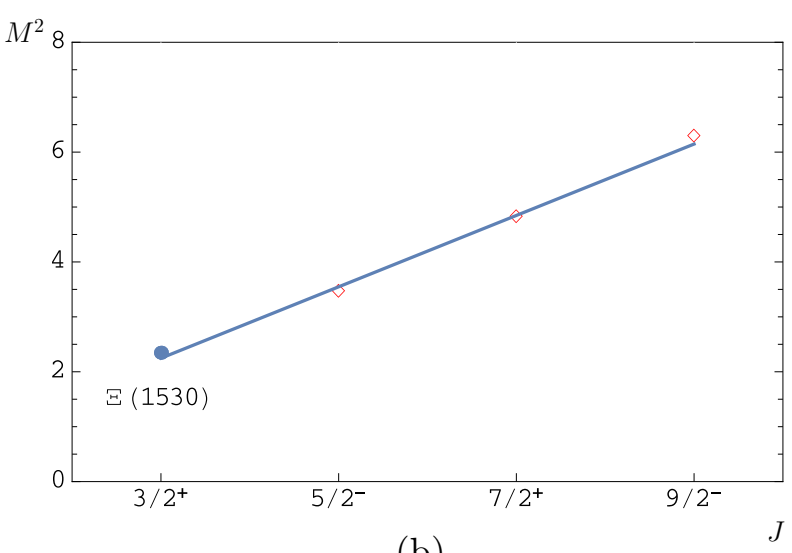

(b)

FIG. 3: Same as in Fig. 1 for the $\Xi$ baryons.

of light mesons and charmed baryons. Moreover they follow the same pattern as the slopes of heavy baryons: the slope decreases with the increase of the diquark mass as well as with the increase of the parent baryon mass.

\section{CONCLUSIONS}

The mass spectra of strange baryons were calculated in the framework of the relativistic quark model based on the quasipotential approach. The quark-diquark picture, which had been previously successfully applied for the investigation of the spectroscopy of heavy baryons [13, 14], was extended to the strange baryons. Such approach allows one to reduce very complicated relativistic three-body problem to the subsequent solutions of two two-body problems. It is assumed that the baryon is the bound quark-diquark system, where two quarks with equal constituent masses form a diquark. The diquarks are not treated to be the point-like objects. Instead their internal structure is taken into account by the introduction of the form factors expressed in terms of the diquark wave functions. The diquark masses and form factors were calculated using the solutions of the relativistic quasipotential equation with the kernel which nonperturbatively accounts for the relativistic effects. It was found that for the correct description of the strange baryon mass spectra it is necessary to consider not only the ground state scalar and axial vector diquarks, as we did in our previous study of heavy baryon spectroscopy [14], but also their first orbital and radial excitations. The ground state and excited baryon masses were obtained by solving the relativistic quark-diquark quasipotential equation. Note that in our analysis we did not make any new assumptions about the quark interaction in baryons or introduce any new parameters. The values of all parameters were taken from previous considerations of meson properties. This significantly increases the reliability and predictive power of our approach. The masses of strange baryons were calculated up to rather high orbital and radial excitations. This allowed us to construct the Regge trajectories which were found to be linear. The validity of the proposed relations between the Regge slopes and intercepts was tested.

The obtained results were compared with available experimental data [1] and previous predictions within different theoretical approaches [20 24]. We found that all 4- and 3-star states of strange baryons with established quantum numbers are well reproduced in our model as well as most of the 2- and 1-star states. Possible candidates for the experimentally 
observed states with unknown quantum numbers can be identified. We emphasize that the experimental mass of the $\Lambda(1405)$ is naturally reproduced in our model, while its rather low mass presents some difficulties for most of the three-quark models [20 22]. It is necessary to note that our quark-diquark picture predicts less excited states of strange baryons than the three-body approaches. The distinctions become apparent for higher baryon excitations. However the number of predicted strange baryon states still significantly exceeds the number of observed ones. Thus the experimental determination of the quantum numbers of the already observed $\Xi$ and $\Omega$ excited states as well as the further search for the missing excited states of strange baryons represents highly promising and important problem.

\section{Acknowledgments}

The authors are grateful to D. Ebert, V. A. Matveev and V. I. Savrin for useful discussions.

[1] K.A. Olive et al. (Particle Data Group), Chin. Phys. C, 38, 090001 (2014).

[2] N. Brambilla et al., Eur. Phys. J. C 71, 1534 (2011).

[3] L. Maiani, F. Piccinini, A. D. Polosa and V. Riquer, Phys. Rev. D 71, 014028 (2005); L. Maiani, F. Piccinini, A. D. Polosa and V. Riquer, Phys. Rev. D 89, no. 11, 114010 (2014).

[4] D. Ebert, R. N. Faustov and V. O. Galkin, Phys. Lett. B 634, 214 (2006); Eur. Phys. J. C 58, 399 (2008).

[5] R. L. Jaffe, Phys. Rep. 409, 1 (2005).

[6] G. 't Hooft, G. Isidori, L. Maiani, A. D. Polosa and V. Riquer, Phys. Lett. B 662, 424 (2008);

L. Maiani, F. Piccinini, A. D. Polosa and V. Riquer, Phys. Rev. Lett. 93, 212002 (2004).

[7] D. Ebert, R. N. Faustov and V. O. Galkin, Eur. Phys. J. C 60, 273 (2009).

[8] E. Klempt and J. M. Richard, Rev. Mod. Phys. 82, 1095 (2010).

[9] V. Crede and W. Roberts, Rept. Prog. Phys. 76, 076301 (2013).

[10] A. V. Anisovich, V. V. Anisovich, M. A. Matveev, V. A. Nikonov, A. V. Sarantsev and T. O. Vulfs, Int. J. Mod. Phys. A 25, 2965 (2010) [Int. J. Mod. Phys. A 25, 3155 (2010)].

[11] H. Forkel and E. Klempt, Phys. Lett. B 679, 77 (2009).

[12] D. Ebert, R. N. Faustov, V. O. Galkin and A. P. Martynenko, Phys. Rev. D 66, 014008 (2002).

[13] D. Ebert, R. N. Faustov and V. O. Galkin, Phys. Rev. D 72, 034026 (2005); Phys. Lett. B 659, 612 (2008).

[14] D. Ebert, R. N. Faustov and V. O. Galkin, Phys. Rev. D 84, 014025 (2011).

[15] D. Ebert, R. N. Faustov, V. O. Galkin and A. P. Martynenko, Phys. Rev. D 70, 014018 (2004) [Phys. Rev. D 77, 079903 (2008)].

[16] D. Ebert, R. N. Faustov and V. O. Galkin, Phys. Rev. D 73, 094002 (2006).

[17] D. Ebert, V. O. Galkin and R. N. Faustov, Phys. Rev. D 57, 5663 (1998) [Phys. Rev. D 59, 019902 (1999)]; Phys. Rev. D 67, 014027 (2003).

[18] A.M. Badalian, A.I. Veselov and B.L.G. Bakker, Phys. Rev. D 70, 016007 (2004); Yu.A. Simonov, Phys. Atom. Nucl. 58, 107 (1995).

[19] D. Ebert, R. N. Faustov and V. O. Galkin, Phys. Rev. D 79, 114029 (2009).

[20] S. Capstick and N. Isgur, Phys. Rev. D 34, 2809 (1986).

[21] U. Loring, B. C. Metsch and H. R. Petry, Eur. Phys. J. A 10, 447 (2001). 
[22] T. Melde, W. Plessas and B. Sengl, Phys. Rev. D 77, 114002 (2008).

[23] E. Santopinto and J. Ferretti, arXiv:1412.7571 [nucl-th].

[24] G. P. Engel et al. [BGR Collaboration], Phys. Rev. D 87, no. 7, 074504 (2013).

[25] A. B. Kaidalov, Z. Phys. C 12, 63 (1982).

[26] L. Burakovsky and J. T. Goldman, Phys. Lett. B 434, 251 (1998).

[27] X. H. Guo, K. W. Wei and X. H. Wu, Phys. Rev. D 78, 056005 (2008). 